\title{
Implications for reflective practice and safer care in paramedicine: the Bawa-Garba case.
}

\author{
Published as: Delport, S., Gyuran, J., Knox, S., Batt, A.M. Implications for reflective practice \\ and safer care in paramedicine: the Bawa-Garba case. Journal of Paramedic Practice. \\ 2018;10(11):462. 10.12968/jpar.2018.10.11.462
}

\section{Dear Editor,}

Your readers may be aware of the Bawa-Garba case in the UK. The High Court ruled, in January 2018, that Hadiza Bawa-Garba, a paediatrics trainee (resident), was to be struck off the General Medical Council (GMC) register in the UK (England and Wales High Court (EWHC), 2018). She was previously convicted of gross negligence manslaughter in 2015 after the death of a 6-year-old boy from sepsis at Leicester Royal Infirmary (Dyer, 2015). A nurse involved in the case was also previously convicted, and struck off the Register of Nurses and Midwives. The consultant with overall clinical responsibility has faced no investigation or sanctions.

There were certainly very serious errors made by Bawa-Garba in the clinical assessment and management of this case (EWHC, 2018). However, the report that resulted from the clinical incident review after the death, also stated that many of the events leading to the boy's death were system failings, which included understaffing, use of agency staff, IT systems failure, demanding workload, and shift scheduling. It included recommendations to improve support for trainees, and enhance patient safety. This report was seemingly not considered in the court case that resulted in the conviction of Bawa-Garba.

Disturbingly, a reflective entry from Bawa-Garba's e-portfolio, which she was asked to complete 7 days after the incident, was considered in the case. Surely, a document in which trainees reflect with the aim to learn from their mistakes should not be used in punitive action against them - even more so, in light of significant system failings? When blame becomes the focus of proceedings, fear and humiliation make safer care more difficult as a result of decreased reporting of incidents (Robinson, 2002). A system which blames trainees for outcomes in light of system issues, is one that is destined to hamper future trainee development. Fixing systems issues and using a just culture approach, rather than punishing or firing staff, is the route to creating safe systems.

The ruling has broader implications than those for the parties directly involved. Recently, physician educators in the UK have stated that they are seeing significant changes in how trainees reflect on their practice. This ruling may lead to a lack of honest reflection by trainees, or worse, the cover-up of mistakes and errors. The Medical Protection Society (MPS) has also stated that this ruling could 'jeopardise an open, learning culture in health care at a time when the profession is already marred by low morale and fear (MPS, 2018).'

Reflective practice has been encouraged within the paramedic profession as a method used to demonstrate competence and professionalism (O'Meara, 2009; Trede, 2009). 'Paramedics are responsible for reassuring the public and their colleagues of their clinical competence through undertaking a number of activities, these include... self and peer review and reflective practice' (Woollard, 2009). Indeed, some paramedic regulators insist on reflective practice as an integral component of the registrant's portfolio to support evidence of competence and continued registration (Pre-Hospital Emergency Care Council (PHECC), 2013). 
As committed proponents of reflective practice in paramedicine, this case certainly makes us uneasy. We sincerely hope that this does not set a precedent with paramedic regulators, or result in negative consequences for the role of reflection in paramedic practice.

Yours,

Shannon Delport, Senior Lecturer, CQUniversity, Queensland, Australia

John Gyuran, Paramedic Educator, Centre for Paramedic Education and Research, Ontario, Canada

Shane Knox, Adjunct Clinical Professor, University College Cork, Ireland

Alan M. Batt, Adjunct Associate Professor, CQUniversity, Queensland, Australia

\section{References}

Dyer C. Paediatrician found guilty of manslaughter after boy's death from septic shock. Br Med J. 2015; 351

England and Wales High Court [(Administrative Court) Decisions]. HC76 (Admin): General Medical Council v. Dr Bawa-Garba. British and Irish Legal Information Institute; 2018. https://tinyurl.com/yatxpz54 (accessed 28 October 2018)

Pre-Hospital Emergency Care Council. Continuous Professional Competence: A guide for Emergency Medical Technicians registered with the Pre-Hospital Emergency Care Council. Naas, Ireland: PHECC; 2013

Medical Protection Society. MPS response to judgement on the case of Dr Bawa-Garba vs GMC. Press Releases. 2018. https://tinyurl. com/ybpvlz2f (accessed 28 October 2018)

O'Meara P. Paramedics marching towards professionalism. Journal of Emergency Primary Health Care. 2009; 7(1): article 990339.

Robinson K. "To err is human...." patient safety initiatives for EMS. J Emerg Nurs. 2002; 28(1):47-48

Trede F. Becoming professional in the 21st century. Australasian J Paramed. 2009; 7(4) Woollard M. Professionalism in the UK paramedic practice. J Emerg Primary Health Care. $2009 ; 7(4)$ 